\title{
Oxaliplatin in pre-treated patients: maybe not the match point?
}

\author{
Chiara Caparello $^{1}$, Enrico Vasile ${ }^{1}$, Ingrid Garajova ${ }^{2}$, Asif Ali $^{3,4}$, Elisa Giovannetti ${ }^{5,6}$ \\ ${ }^{1}$ Department of Medical Oncology, University Hospital of Pisa, Pisa, Italy; ${ }^{2}$ Department of Experimental, Diagnostic and Specialty Medicine, \\ University of Bologna, Bologna, Italy; ${ }^{3}$ Institute of Basic Medical Sciences, Khyber Medical University, Peshawar, Pakistan; ${ }^{4}$ Institute of Cancer \\ Sciences, College of Medical, Veterinary and Life Sciences, University of Glasgow, Scotland, UK; ${ }^{5}$ Department of Medical Oncology, VU University \\ Medical Center, Amsterdam, The Netherlands; ${ }^{6}$ Cancer Pharmacology Lab, AIRC Start-Up Unit, Department Translational Medicine, University of \\ Pisa, Pisa, Italy \\ Correspondence to: Elisa Giovannetti, MD, PhD. Associate Professor and Clinical Pharmacologist, Lab Medical Oncology, Cancer Center Amsterdam, \\ VU University Medical Center, De Boelelaan 1117, 1081HV Amsterdam, The Netherlands. Email: elisa.giovannetti@gmail.com. \\ Comment on: Gill S, Ko YJ, Cripps C, et al. PANCREOX: A Randomized Phase III Study of 5-Fluorouracil/Leucovorin With or Without Oxaliplatin \\ for Second-Line Advanced Pancreatic Cancer in Patients Who Have Received Gemcitabine-Based Chemotherapy. J Clin Oncol 2016. [Epub ahead \\ of print].
}

\begin{abstract}
Pancreatic cancer is an aggressive disease and is projected to become one of the major causes of cancer-related death in western countries. Intensification of first-line treatment with the use of polychemotherapeutic regimens (FOLFIRINOX, gemcitabine plus nab-paclitaxel) has significantly improved the overall survival and progression-free survival; as a consequence, the number of patients alive and fit for second-line treatment is increasing. However, the selection of the best second-line treatment is difficult and large phase III trials are urgently needed. After the positive results of oxaliplatin and fluorouracil combination in the CONKO-003 trial, two recent phase III studies, PANCREOX and NAPOLI-1, investigating the use of second-line chemotherapy after gemcitabine-based first-line, showed controversial results. Giving the practice-changing advances rapidly growing in last years and the multiple therapeutic strategies that are becoming available for the treatment of pancreatic cancer, the real issue seems to be the optimal sequence of treatment much more than second-line treatment.
\end{abstract}

Keywords: Pancreatic cancer; oxaliplatin; metastatic patients; second-line treatment; need of better patients selection

Submitted Dec 05, 2016. Accepted for publication Dec 07, 2016.

doi: $10.21037 /$ tcr.2017.01.35

View this article at: http://dx.doi.org/10.21037/tcr.2017.01.35

\section{PANCREOX trial and second-line treatment after gemcitabine-based first-line}

Pancreatic cancer is an extremely aggressive disease, projected to become the second leading cause of cancerrelated death (1). While the attempt of using a personalized approach in first line setting failed, the intensification of the treatment, by the use of the combination of gemcitabine and nanoparticle albumin-bound paclitaxel (nab-paclitaxel) and the triplet regimen FOLFIRINOX (a combination of 5-fluorouracil (5-FU), leucovorin, irinotecan and oxaliplatin) have significantly improved the outcome of these patients (2-4).

Given these results, the number of patients fit for a second-line treatment is significantly increasing and the identification of a standard second-line approach is urgently needed. Currently, there is no standard of treatment after FOLFIRINOX; in the French phase III study 80 patients in the FOLFIRINOX group and 85 patients in the gemcitabine group received a second-line treatment. In particular, in the "FOLFIRINOX group", gemcitabine or a gemcitabine-based combination were administered in the majority of patients; whereas in the "gemcitabine group" most patients were treated with combinations containing oxaliplatin or cisplatin (2). However, no difference in overall survival was noted between the two groups (i.e., 4.4 months each).

Data about the use of oxaliplatin in second-line setting 
derive from the results of the German CONKO-003 phase III randomized trial, firstly presented in 2008 , and then with updated results in 2014 (5). This phase III trial was initially designed to compare folinic acid, fluorouracil administered weekly in 24-hour infusion for the first 4 weeks and oxaliplatin administered on days 8,22 of a 6 -week-cycle (OFF) versus best supportive care. However, because of the initial data about second-line treatment derived from phase II studies, the trial was modified and patients were randomly assigned to receive OFF or folinic acid and fluorouracil (FF). OFF resulted in a statistically significant increase in overall survival (5.9 vs. 3.3 months; $\mathrm{P}=0.010$ ) and in progressionfree survival ( 2.9 vs. 2.0 months; $\mathrm{P}=0.019$ ). The toxicity profile was not significantly different between the two arms and both FF and OFF resulted feasible in this pre-treated population, with the exception of grade 3 neuropathy that was present in three patients in OFF treatment while absent in $\mathrm{FF}$ treatment (5). Of note, only one patient in $\mathrm{OFF}$ arm experienced grade 3 thrombocytopenia, while none experienced grade 3 leucopenia; thus, $81 \%$ of oxaliplatin administrations were full dose.

More recently, the PANCREOX trial has evaluated the potential advantage of the more widely used combination containing oxaliplatin and 5-fluorouracil as a bolus and in continuous infusion (m-FOLFOX 6 regimen) versus 5 -fluorouracil monotherapy (6), trying to confirm the role of oxaliplatin in the second-line setting. This study was closed after the enrollment of 108 patients before the target accrual of 128 patients; after a median follow-up of 8.8 months no difference in progression-free survival (3.1 vs. 2.9 months; $\mathrm{P}=0.989)$ was observed between the two arms, while, the median overall survival (OS) was lower in the m-FOLFOX6 arm than in the 5-fluorouracil alone arm (6.1 vs. 9.9 months; $\mathrm{P}=0.024$ ).

The conflicting results from the CONKO-003 and the PANCREOX trials seem to undermine the role of oxaliplatin in pre-treated patients, but several important aspects of these trials should be considered more carefully. The most evident bias possibly affecting the results in PANCREOX trial is the adherence to the treatment: an overall incidence of grade 3-4 adverse events of $63 \%$ in FOLFOX arm can actually affect the results: $10 \%$ of permanent discontinuation was registered in this arm comparing to $0 \%$ of $\mathrm{FU}$ alone and almost half of the patients needed a dose reduction of oxaliplatin and 5 -fluorouracil due to hematologic toxicity or neuropathy. Remarkably, the patients randomized in the FOLFOX arm had worse general conditions than the patients treated with 5-fluorouracil one (11.1\% vs. 5.7\% had ECOG PS 2), however, patients treated with FOLFOX had a longer median time since the diagnosis of advanced disease $(7.9 \mathrm{vs}$. 5.7 months).

Another interesting point is that a study withdrawal due to disease progression was more frequent in the 5 -fluorouracil/leucovorin arm $(75 \%$ vs. $55 \%)$ while the cause was the development of adverse events in $20 \%$ of the patients treated with FOLFOX, versus $2 \%$ in patients treated with 5 -fluorouracil/leucovorin.

Furthermore, among post-progression treatments, aggressive combinatory treatments were more frequently used after 5-fluorouracil/leucovorin than after FOLFOX. These therapeutic choices could have been influenced by adverse events experienced by patients and performance status at progressive disease. This might at least in part explain the overall survival in the 5-fluorouracil/leucovorin arm, which is extraordinarily long for second-line setting (i.e., 9.9 months).

Although comparisons between OFF (5) and PANCREOX studies can be only speculative and not made according to correct statistical methodology, some additional considerations should be taken into account, as follows. Both of these trials were designed in the "gemcitabine-monotherapy era", before the revolution given by FOLFIRINOX triplet regimen and gemcitabine and nab-paclitaxel doublet; only in the PANCREOX trial a proportion $(25.9 \%$ in FOLFOX arm and $22.2 \%$ in the 5 -fluorouracil/leucovorin arm) of the patients had been treated with gemcitabine-based combinations, while in CONKO-003 trial all the patients had received gemcitabine monotherapy in first-line setting.

The most evident difference between the two trials is in the regimen used: the increased oxaliplatin intensity and the presence of the 5-fluorouracil bolus in PANCREOX trial may have played an important role in the results, suggesting a role of oxaliplatin in pre-treated patients, within an acceptable modulated schedule.

The use of oxaliplatin in second-line setting was also evaluated in a phase II Japanese study: patients were randomly assigned to receive S-1 monotherapy or S-1 in combination with oxaliplatin (SOX) after progression on a gemcitabine-based first-line chemotherapy. Despite the increase in response rate, only a trend in favour of SOX was observed in progression-free survival and overall survival (7).

Most recently, the NAPOLI-1 phase III trial enlarged the perspective of second-line treatment in gemcitabinepretreated patients. This was a large, multicenter trial, enrolling more than 400 patients, designed with three 
Table 1 Main characteristic of trials CONKO-003, PANCREOX, NAPOLI-1

\begin{tabular}{llll}
\hline Characteristic & CONKO-003 & PANCREOX & NAPOLI-1 \\
\hline No. of patients & $168(77$ OFF/91 FF) & 108 (54 mFOLFOX6/54 FU/LV) & 417 (151 NAL-IRI*/149 FU/FA**/117 NAL-IRI + FU/FA) \\
Regimens & OFF; FF & mFOLFOX-6; FU/LV & NAL-IRI + FU/FA; FU/FA; NAL-IRI \\
Overall survival & 5.9 months; 3.3 months & 6.1 months; 9.9 months & 6.1 months; 4.2 months; 4.9 months \\
\hline
\end{tabular}

FF, folinic acid and fluorouracil; FOLFOX, folinic acid (leucovorin), 5-fluorouracil and oxaliplatin; FU/FA, 5-fluororacil and folinic acid; FU/LV, 5-fluororacil and leucovorin; NAL-IRI, nanoliposomal irinotecan; OFF, oxaliplatin, 5-fluorouracil and folinic acid. *, nanoliposomal irinotecan; ${ }^{\star \star}, 5$-fluororacil and folinic acid.

arms: nanoliposomal irinotecan plus fluorouracil and folinic acid, nanoliposomal irinotecan monotherapy or fluorouracil monotherapy (i.e., the same control arm as in the CONKO.003 trial). Patients had received gemcitabinebased regimens also in neoadjuvant and adjuvant setting (if progressed within 6 months) and in advanced disease. Moreover, patients were selected for toxicity by the evaluation of UGTA1 genotype testing; the initial dose was reduced in case of homozygous result.

Since the study was initially designed to have only two arms (nanoliposomal irinotecan monotherapy or 5 -fluorouracil monotherapy) and then amended to have three arms, a total of 151 patients were treated with nanoliposomal monotherapy, 149 patients with 5-fluorouracil and folinic acid and 117 patients with the combination, respectively. The primary endpoint was met: median overall survival was 6.1 months in the nanoliposomal irinotecan and 5-fluorouracil combination arm and 4.2 months in the fluorouracil and folinic acid arm $(\mathrm{P}=0.012)$, while overall survival was 4.9 months for patients treated with nanoliposomal irinotecan alone, demonstrating a not significant difference versus the 5 -fluorouracil arm. This study suggested the prognostic role of several factors, including factors reported in the first-line setting, such as performance status, liver metastases, albumin levels and baseline CA 19.9 value (2,3). Of note, patients with adverse prognostic factors allocated in the combination arm had lower hazard ratio than patients who received 5-fluorouracil, suggesting the use of a more aggressive treatment in this subgroup of patients.

Remarkably, most of the secondary endpoints were met: a statistically significant difference was indeed reported in progression-free survival, time-to-treatment failure, response rate and CA 19.9 decrease. This new treatment can therefore be considered a new therapeutic option in second-line setting in patients pre-treated with gemcitabine or gemcitabinebased combination, but some important consideration should be done. Firstly, the combination treatment showed a higher rate of grade 3 or 4 neutropenia: $27 \%$ versus $15 \%$ of nanoliposomal irinotecan monotherapy and $1 \%$ of 5 -fluorouracil monotherapy. Furthermore, $3 \%$ of the patients in the combination arm experienced neutropenic sepsis.

The choice of 5-fluorouracil as control arm has been correct from a methodological point of view, although its current use in clinical practice, with patients with good performance status, suggests a diffuse use of combinations in second-line setting (8-10). However, the use of the same control as in the CONKO-003 trial makes some indirect comparisons possible. The control arm performed better in this trial than in CONKO-003 with regards to overall survival (4.2 vs. 3.3 months), and in both cases these data were reliable and consistent with data derived from literature. Still, the survival reported for experimental arm in these trials was almost superimposable: 6.1 months in the nanoliposomal irinotecan and 5-fluorouracil and 5.9 months in the OFF combination, respectively.

Table 1 summarizes the main characteristic of the trials CONKO-003, PANCREOX and NAPOLI-1. Of note, the selection criteria of the patients in these studies are quite different: in the CONKO-003 trial the patients were all treated in first line treatment for advanced disease (either locally advanced or metastatic), while in the NAPOLI-1 trial also patients progressed within 6 months from adjuvant/neoadjuvant treatment were included. So, if on one hand we can say that in the NAPOLI- 1 trial all the patients were metastatic at the time of randomization, while in the CONKO-003 about $12 \%$ of patients were locally advanced, on the other hand the setting of treatment of the previous gemcitabine-based therapy was not specified in NAPOLI-1 trial leading to enrollment of a relatively not homogeneous population including patients progressed after an adjuvant treatment and patients largely pretreated in the metastatic setting (about 30\% of patients had indeed received 2 or more lines for metastatic disease). 
Taking into account the current use of nab-paclitaxel in first-line setting and the neurotoxicity that derives from this therapy, the use of nanoliposomal irinotecan in secondline setting seems to have less neurological side effects than oxaliplatin, however, the hematologic toxicity was not negligible in the NAPOLI- 1 trial.

Finally, data about the use of irinotecan or FOLFIRI regimen derive from little, prospective or retrospective reports, and at today no phase III trial has investigated this regimen in this setting (11-15).

\section{Second-line treatment after FOLFIRINOX first-line}

There is currently no standard in second-line treatment for patients progressed on the triplet regimen. In the French phase III trial 80 patients in the "FOLFIRINOX group" and 85 patients in the "gemcitabine group" underwent a second-line treatment. In particular, in the FOLFIRINOX arm, gemcitabine (in $82.5 \%$ of cases) or a gemcitabinebased combination (in $12.5 \%$ ) were used as a second-line treatment (2), but no difference in second-line OS was noted between the two arms (i.e., 4.4 months each).

Several retrospective series evaluated the use of a secondline treatment with no conclusive results (8-10). A prospective French trial enrolled patients to receive gemcitabine and nabpaclitaxel as second-line treatment after FOLFIRINOX with encouraging results: 57 patients were treated, obtaining a disease control rate of $58 \%$, and an objective response rate of $17.5 \%$. Median overall survival (OS) was 8.8 months and median progression-free survival was 5.1 months (16). Other data about the use of gemcitabine and nab-paclitaxel combination derive from retrospective reports $(17,18)$. Prospective phase III trials are therefore urgently needed for the identification of the standard second-line treatment after the triplet-drug regimen.

\section{Future perspectives}

Considering the latest news in the second-line treatment and the results of CONKO-003, NAPOLI- 1 and PANCREOX trials, further studies should evaluate the role of the combination of 5 -fluorouracil and oxaliplatin in second-line setting in a larger, multicenter study, taking into account the new standard regimens in first-line treatment and using a more feasible schedule. However, the availability of two main options in first-line setting, deriving from phase III randomized trials, without direct comparisons between FOLFIRINOX or modified schedules and gemcitabine-nab-paclitaxel combination, makes the choice of second-line treatment more difficult, depending mostly on the choice of first-line.

No predictive factors of response at today can help clinicians in the choice of first-line treatments, which is actually based on clinical, patient or disease-related, factors. Biomarkers that might guide these therapies are urgently warranted and might be useful also in the second-line setting (19).

Furthermore, given all the recent diagnostics and prognostics improvements in a disease that had almost no treatment available until few years ago, the real issue is not only the search for the best second-line treatment, but, the identification of the optimal sequence of treatment, considering the aggressive and rapidly progressing nature of pancreatic cancer.

Finally, in the design of possible randomized studies aimed at the individuation of the ideal sequence-strategy, it should be taken into account the further change of the landscape that might derive from ongoing trials evaluating the triplet regimen FOLFIRINOX, FOLFOXIRI and the combination of gemcitabine and nab-paclitaxel in the adjuvant setting (20-22).

\section{Acknowledgments}

The authors would like to thank the Italian Association for Cancer Research (AIRC), Istituto Toscano Tumori (ITT), and Cancer Center Amsterdam (CCA) Foundation.

Funding: None

\section{Footnote}

Provenance and Peer Review: This article was commissioned and reviewed by the Section Editor Lei Wen (Department of Clinical Oncology Xijing Hospital, the Fourth Military Medical University, Xi'an, China).

Conflicts of Interest: All authors have completed the ICMJE uniform disclosure form (available at http://dx.doi. org/10.21037/tcr.2017.01.35). The authors have no conflicts of interest to declare.

Ethical Statement: The authors are accountable for all aspects of the work in ensuring that questions related to the accuracy or integrity of any part of the work are appropriately investigated and resolved.

Open Access Statement: This is an Open Access article distributed in accordance with the Creative Commons Attribution-NonCommercial-NoDerivs 4.0 International License (CC BY-NC-ND 4.0), which permits the non- 
commercial replication and distribution of the article with the strict proviso that no changes or edits are made and the original work is properly cited (including links to both the formal publication through the relevant DOI and the license). See: https://creativecommons.org/licenses/by-nc-nd/4.0/.

\section{References}

1. Siegel RL, Miller KD, Jemal A. Cancer statistics, 2016. CA Cancer J Clin 2016;66:7-30.

2. Conroy T, Desseigne F, Ychou $M$, et al. FOLFIRINOX versus gemcitabine for metastatic pancreatic cancer. $\mathrm{N}$ Engl J Med 2011;364:1817-25.

3. Von Hoff DD, Ervin T, Arena FP, et al. Increased survival in pancreatic cancer with nab-paclitaxel plus gemcitabine. N Engl J Med 2013;369:1691-703.

4. Singhal MK, Kapoor A, Bagri PK, et al. A phase III trial comparing folfirinox versus gemcitabine for metastatic pancreatic cancer. Ann Oncol 2014;25:iv210-iv211.

5. Oettle H, Riess H, Stieler JM, et al. Second-line oxaliplatin, folinic acid, and fluorouracil versus folinic acid and fluorouracil alone for gemcitabine-refractory pancreatic cancer: outcomes from the CONKO-003 trial. J Clin Oncol 2014;32:2423-9.

6. Gill S, Ko YJ, Cripps C, et al. PANCREOX: A Randomized Phase III Study of 5-Fluorouracil/Leucovorin With or Without Oxaliplatin for Second-Line Advanced Pancreatic Cancer in Patients Who Have Received Gemcitabine-Based Chemotherapy. J Clin Oncol 2016. [Epub ahead of print].

7. Ohkawa S, Okusaka T, Isayama H, et al. Randomised phase II trial of S-1 plus oxaliplatin vs S-1 in patients with gemcitabine-refractory pancreatic cancer. Br J Cancer 2015;112:1428-34.

8. Caparello C, Vivaldi C, Fornaro L, et al. Second-line therapy for advanced pancreatic cancer: evaluation of prognostic factors and review of current literature. Future Oncol 2016;12:901-8.

9. Pellei C, Lanese A, Bittoni A, et al. Second-line treatment in patients with FOLFIRINOX-refractory pancreatic adenocarcinoma (PDAC): Doublets or single-agent chemotherapy? J Clin Oncol 2014;32:abstr e15186.

10. Ducoulombier A, Quintin J, Desjardin M, et al. Growth modulation index (GMI) to assess salvage chemotherapy benefit after FOLFIRINOX progression in metastatic pancreatic adenocarcinoma. Eur J Cancer 2015;51:S430.

11. Cereda S, Reni M, Rognone A, et al. XELIRI or FOLFIRI as salvage therapy in advanced pancreatic cancer. Anticancer Res 2010;30:4785-90.

12. Zaniboni A, Aitini E, Barni S, et al. FOLFIRI as secondline chemotherapy for advanced pancreatic cancer: a
GISCAD multicenter phase II study. Cancer Chemother Pharmacol 2012;69:1641-5.

13. Yoo C, Hwang JY, Kim JE, et al. A randomised phase II study of modified FOLFIRI. 3 vs modified FOLFOX as second-line therapy in patients with gemcitabine-refractory advanced pancreatic cancer. Br J Cancer 2009;101:1658-63.

14. Gebbia V, Maiello E, Giuliani F, et al. Irinotecan plus bolus/infusional 5-Fluorouracil and leucovorin in patients with pretreated advanced pancreatic carcinoma: a multicenter experience of the Gruppo Oncologico Italia Meridionale. Am J Clin Oncol 2010;33:461-4.

15. Neuzillet C, Hentic O, Rousseau B, et al. FOLFIRI regimen in metastatic pancreatic adenocarcinoma resistant to gemcitabine and platinum-salts. World J Gastroenterol 2012;18:4533-41.

16. Portal A, Pernot S, Tougeron D, et al. Nab-paclitaxel plus gemcitabine for metastatic pancreatic adenocarcinoma after Folfirinox failure: an AGEO prospective multicentre cohort. Br J Cancer 2015;113:989-95.

17. Zhang Y, Hochster HS, Stein S, et al. Second-line gemcitabine plus nab-paclitaxel $(\mathrm{G}+\mathrm{A})$ for advanced pancreatic cancer (APC) after first-line FOLFIRINOX: Single institution retrospective review of efficacy and toxicity. J Clin Oncol 2014;32:abstr 344.

18. Salem ME, Alistar AT, Dyson G, et al. Albumin-bound paclitaxel plus gemcitabine after first-line FOLFIRINOX therapy in patients with pancreatic cancer. J Clin Oncol 2014;32:abstr e15252.

19. Caparello C, Meijer LL, Garajova I, et al. FOLFIRINOX and translational studies: Towards personalized therapy in pancreatic cancer. World J Gastroenterol 2016;22:6987-7005.

20. Nab-paclitaxel and Gemcitabine vs. Gemcitabine Alone as Adjuvant Therapy for Patients With Resected Pancreatic Cancer. ClinicalTrials.gov NCT01964430. (This study is ongoing, but not recruiting participants).

21. Italian Multicenter Study Comparing FOLFOXIRI Versus Gemcitabine as Adjuvant Treatment for Resected Pancreatic Cancer. ClinicalTrials.gov NCT02355119. (This study is currently recruiting participants).

22. Trial Comparing Adjuvant Chemotherapy With Gemcitabine Versus mFolfirinox to Treat Resected Pancreatic Adenocarcinoma. ClinicalTrials.gov NCT01526135. (This study is currently recruiting participants).

Cite this article as: Caparello C, Vasile E, Garajova I, Ali A, Giovannetti E. Oxaliplatin in pre-treated patients: maybe not the match point? Transl Cancer Res 2017;6(Suppl 1):S163-S167. doi: $10.21037 /$ tcr.2017.01.35 